\title{
Corela
}

Cognition, représentation, langage

HS-29 | 2019

Questions et exclamations au prisme de plusieurs approches linguistiques

\section{Les questions en anglais d'un point de vue diachronique}

Sylvie HANCIL

(2) OpenEdition

Journals

Édition électronique

URL : http://journals.openedition.org/corela/8945

DOI : $10.4000 /$ corela.8945

ISSN : 1638-573X

Éditeur

Cercle linguistique du Centre et de l'Ouest - CerLICO

Référence électronique

Sylvie HANCIL, "Les questions en anglais d'un point de vue diachronique », Corela [En ligne], HS-29

2019, mis en ligne le 09 septembre 2019, consulté le 30 octobre 2019. URL : http://

journals.openedition.org/corela/8945; DOI : 10.4000/corela.8945

Ce document a été généré automatiquement le 30 octobre 2019

\section{cc) (i) (9) (-)}

Corela - cognition, représentation, langage est mis à disposition selon les termes de la licence

Creative Commons Attribution - Pas d'Utilisation Commerciale - Partage dans les Mêmes Conditions

4.0 International. 


\title{
Les questions en anglais d'un point de vue diachronique
}

\author{
Sylvie HANCIL
}

\section{Introduction}

1 Dans cet article sur les questions dans l'histoire de l'anglais, on se propose de discuter cinq points d'après les exemples donnés dans le texte en anglais contemporain. Après avoir présenté l'état des lieux depuis le vieil-anglais, on expliquera comment les pronoms relatifs en wh- se sont introduits, on discutera des raisons qui expliquent l'apparition de do, puis on complètera cette étude par une analyse du statut informationnel des syntagmes nominaux thématisés et des prédications existentielles. On s'intéressera aussi aux questions tags en diachronie et on finira par la grammaticalisation des questions fermées.

\section{Les faits}

2 Le but de cette première partie est de montrer que les questions directes et indirectes existaient depuis le vieil-anglais.

\subsection{Les faits en vieil-anglais}

3 Il y a en vieil-anglais deux types de questions: les questions fermées ('yes-no interrogatives', dont un exemple en anglais contemporain se trouve 1.21 dans le texte proposé) sont typiquement des phrases à l'indicatif avec l'ordre verbe-sujet (exemple (1)). Cependant, elles peuvent être introduites par hwceper 'whether'(exemple (2)) : ce type de questions n'a pas d'inversion du sujet. Les questions ouvertes ('contentquestions', dont un exemple en anglais contemporain est 1.6 dans le texte), quant à elles, contiennent un pronom interrogatif comme hwcet 'what', hwa 'who', hwelc 'which', $h u$ 'how', hwoer 'where', hwceðer 'which of the two' (exemples (3) et- (4)). 
(1) Hofst pu cenigne geferan?

Hast thou any companion

'Do you have any companion?'

(IIfric's Colloquy 28)

(2) Hwceðer ge nu secan gold on treowum?

Whether you now seek gold in trees?

'Are you looking for gold in trees?'

(Boethius 32.73.24)

(3) Hwcet getacniað ðonne ða twelf oxan buton ða XII apostolas?

What signify then those twelve oxen except those XII apostles?

'What do those twelve oxen signify other than the twelve apostles?'

(Pastoral Care 16.105.5)

(4) Hwceðer cweðe we ðe ure ðe ðara engla?

Which shall-say we or ours or the angels'?

'Shall we say ours or the angels' (glory) ?'

(Alc. Th. 220.20)

4 Les subordonnées interrogatives en vieil-anglais peuvent appartenir aux deux types de questions : question fermée ( $5 a$ et $b$ ) ou question ouverte (6a et b). Comme en anglais actuel, les questions indirectes respectent l'ordre des mots d'un énoncé déclaratif, c'est-à-dire que le verbe ne bouge pas au-delà du sujet.

(5)

(a) Nyte we hweper se wearmann cefre gefullod

NEG-know we whether the watchman was ever baptized

'we do not know whether the watchman was ever baptized.'

(coaelive, +ALS [Forty-Soldiers] : 293.2671)

(b) peet hi sceoldan secgan hweðer hit soð were

That they should say whether it true was

'that they should say whether it was true'

(coaelive, +ALS [Ash-Wed] : 187.2803)

(6)

(a) pa ludeiscan axodon Crist hwcet he were

The Jews asked Christ what he was

'The Jews asked Christ what he was'

(coaelive, + ALS_ $($ Christmas $)=11.8)$

(b) Sum Iudeisc man wolde gewytan ...hwylce mihte heo hoefde

Some Jewish man would know which power it had

'A Jewish man wanted to know which power it had'

(coaelive, +ALS_ (Basil) : 153.550)

5 Les subordonnées interrogatives peuvent être compléments d'adjectif (7a) et de nom (7b) :

(7)

poet pu sy gemyndig hwoet min foeder pe gedyde

That you be mindful what my father you did

'that you should be mindful of what my father did for you'

(coblick, LS(AssumptMor(BlHom_13)) : 151.245.1887)

(b) Her is on sio swutchung hu Ælfhelm his are

Here is in the declaration how Ælfhelm his property

\& his chta geuadod hoef for Gode \& for wurulde

And his possessions disposed has for God and for world

'Herein is the declaration how Ælfhelm has disposed his property and his possessions for God and for the world'

(codocu3, Ch_1487_(Whitelock_13) :1.220) 


\subsection{Les faits en moyen-anglais}

En moyen-anglais, la question directe se manifeste par l'inversion du sujet et du verbe fini, à moins que, comme en anglais contemporain, le mot en wh- soit le sujet (8-9).

(8) wenst pu pat ic ne cunne singe?

Think you that I not can sing

'Do you think that I can't sing ?'

(The Owl \& Nightingale 47)

(9) Whi seist pou so ?

Why say you so

Why do you say so?

Do est rarement trouvé dans les questions en moyen-anglais ; la première occurrence de la périphrase do est dans Chaucer (10):

(10) Fader, why do ye wepe?

(Canterbury Tales VII.2432)

Whether peut encore être utilisé mais il est suivi d'une inversion de l'ordre des mots (11) :

(11) Whether seistou ( =seist thou) this in ernest or in pley?

(Canterbury Tales I.1125)

Les subordonnées interrogatives sont utilisées dans les mêmes fonctions que les complétives, par exemple comme complément du nom, complément d'objet d'un verbe ou complément d'un adjectif, ou encore sujet. On les trouve après des noms et des prédicats qui sont concernés par la valeur de vérité de la complémentation, comme ask, know, say, wonder, doubt, etc.

10 Whether peut aussi introduire, comme en vieil-anglais, des subordonnées interrogatives mais on peut y trouver le subordonnant 3if. Soit (12-13) :

(12) I noot wher she be womman or goddesse

I not-know whether she is woman or godess

'I don't know if she is a woman or a godess.'

(Canterbury Tales I. 1101)

(13) To telle hir if hir child wente oght forby

'To tell her if her child at all went by there'

(Canterbury Tales VII.600-2)

11 Les subordonnées interrogatives sont introduites par un élément en wh- comme pour les énoncés simples; cet élément peut être un adverbe (where, how, why, etc.) ou un pronom interrogatif. Soit (14-15) :

(14) But sikerly she nyste who was who

'But surely she did not know who was who.'

(CT I.4300)

(15) Who coude wryte which a dedly cheere/Hath Thisbe now, and how hire heer she rente,

'who could write what a deadly expression Thisbe had now, and how she tore her hair.'

(The Legend of Good Women 869-70) 


\subsection{Les faits au début de l'anglais moderne}

12 Au début de l'anglais moderne, les questions sont fréquemment exprimées par des énoncés sans inversion. Les questions de ce type engendrent normalement une réponse affirmative (16).

(16) Wid. You came I thinke from France?

Hel. I did so.

(Shakespeare All's Well that Ends Well III.v) comme belike ou perchance (17)

(17)

Siluia Perchance you think too much of so much pains?

Valentine No (Madam)

(Shakespeare Two Gentlemen of Verona II.i)

$\mathrm{Au}$ début de cette période, les pronoms et adverbes introduisant des questions en whsont presque tous les mêmes qu'en anglais contemporain. La seule exception est whether 'which of the two' (18) :

(18) Laf. Whether doest thou professe thy selfe, a knave or a foole?

Clo. A fool, Sir.

(Shakespeare All's Well that Ends Well IV.v)

moyen-anglais, what était le pronom interrogatif normalement utilisé comme complément d'objet avec des référents animés, même quand l'identité du référent était le sujet de la question. Cet emploi de what peut encore être trouvé dans des textes du 16 ème et du $17^{\text {ème }}$ siècles (19) :

(19) 'Tell me, I prey the,' who was the father of thy childe? She stodyd a whyle, and sayde that it hadde a father. But what was hee?' quoth I. 'Nowe, by my trouth, I knowe not,' quoth shee.

(Holinshed's Chronicle, Harman 69) La périphrase do demeure rare au cours du $15^{\text {ème }}$ siècle. Cependant, au $16^{\text {ème }}$ siècle, on observe une augmentation rapide de l'emploi de do dans les questions (20) qui est parallèle au développement de do dans les énoncés affirmatifs :

(20) What became of the kynge of Castell ... made he ony recovery, or dyd he close hymself in ony of his townes.

Dans la seconde moitié du $16^{\text {ème }}$ siècle, la plupart des questions fermées emploient do. L'inversion est utilisée dans les questions en wh-, là où la périphrase do est d'abord utilisée pour éviter des groupements consonantiques maladroits (21), ou quand un pronom objet inaccentué suit le verbe (22):

(21) What didst thou loose Iacke? (Shakespeare, Henry IV)

(22) What doe you call him ? (Shakespeare, Henry V)

L'emploi de do dans les questions au $18^{\text {ème }}$ siècle est très proche de la norme actuelle. Cependant, l'inversion reste un marqueur de style archaïque (courant dans la Bible du Roi Jacques). De nombreux grammairiens du $18^{\text {ème }}$ siècle rapportent que do peut être omis dans les questions. Cela arrive le plus fréquemment avec des verbes comme know, think, say ou write (23-24).

(23) What say't thou? (Lisle)

(24) In the name of Wonder, Whence came ye? (Farquhar) 


\section{Pronoms démonstratifs et pronoms interrogatifs dans l'histoire de l'anglais}

21 Le deuxième point que j'illustrerai est les relatives en wh- (voir l'exemple en anglais contemporain 1.53 du texte). On s'intéressera à l'introduction des relatifs en wh- à la fin du moyen-anglais et on se demandera s'ils sont la cause ou la conséquence de la disparition des pronoms relatifs se/sepe en vieil-anglais. Je vais donc analyser la distribution des relatifs pronominaux dans les différentes périodes de la langue anglaise en prenant en compte les variables suivantes : 1 . la fonction syntaxique du relatif, qui détermine la progression et la récession des stratégies de relativisation ; 2. le type de subordonnée relative, qu'elle soit restrictive ou non-restrictive.

\section{1. elativisation dans l'histoire de l'anglais}

En vieil-anglais, la stratégie de la relativisation par un pronom relatif est représenté par des membres du paradigme des pronoms démonstratifs se/seo/pœet, qui sont utilisés seuls (25), ou bien accompagnés (26) avec l'adjonction de la particule indéclinable pe:

(25) Đonne cymeð se man se pcet swiftoste hors hafað to pcem cerestan dcele

Then comes the man "Rel." the fastest horse has the first dale

'Then the man who had the fastest horse came to the first dale'

(Q 02/3 NN Hist Ohthr 3 1.20.36)

(26) ac pa men ba pe on woh lifiap, nappap hie na Crist on heora heortan

But those men "Rel." in error live have not they no Christ in their hearts

(Q 02/3 1R Hom Blick6 : 190-1)

A partir du moyen-anglais, les relatifs pronominaux se et sepe coexistent avec d'autres pronoms relatifs, à savoir les éléments en wh-. Le mots en wh-, utilisés en tant que pronoms interrogatifs (27) et relatifs généralisants (28) en vieil-anglais, apparaissent en tant que pronoms relatifs adnominaux au début du moyen-anglais (29), mais n'ont pas un emploi bien établi dans cette fonction avant la fin du moyen-anglais :

(27)

Hwat getacniað ðonne ða twelf oxan buton ða XII apostolas?

What signify then those twelve oxen except those XII apostles?

'What do those twelve oxen signify other than the twelve apostles?'

(Pastoral Care 16.105.5)

(28)

Swa hwa swa syld ceald weeter drincan anum purstigum men para

Whoever gives cold water to drink to-a thirsty man of-those

ðe on ure gelyfað : ne bið his med forloren

"Rel." in us believe not will-be his reward lost

'Whoever gives cold water to drink to one thirsty man of those who believe in me,

his meeds shall not be lost.'

(Ælfric's Homilies I, 38.562.23)

(29)

Đurh ðesse hole mihti and ðurh his gode ileaven was Abraham

Through this holy might and through his good faith was Abraham

iblesced of god, and him behoten Jat on scolde cumen of his kenne

blessed by God and him promised that one should come from his family

ðurh hwam all mankenn scolde bien iblesced

through "Rel." all mankind should be blessed

(QMI IR Relt Vices $1: 109$ ) 
ésumé, la stratégie de la relativisation pronominale a toujours existé en anglais mais les éléments ont changé au cours de l'histoire. Le vieil-anglais et le début du moyen anglais ont des éléments hérités du système démonstratif et à partir du moyenanglais, les relatifs pronominaux sont représentés par des mots en wh-, dérivés des pronoms interrogatifs. En m'appuyant sur la Hiérarchie d'Accessibilité de Keenan et Comrie et les généralisations diachroniques de Maxwell, je montrerai comment les pronoms relatifs en wh- se sont introduits.

\subsection{La Hiérarchie de Keenan \& Comrie et les généralisations diachroniques de Maxwell}

Le Hiérarchie d'Accessibilité de Keenan \& Comrie (1977) est un ordre hiérarchique des positions des syntagmes nominaux qui peuvent être relativisés, en partant du plus accessible vers le moins accessible, c'est-à-dire de la fonction syntaxique la plus fréquemment relativisée à gauche de la hiérarchie à la fonction syntaxique la moins relativisée à droite dans le gradient (30) :

(30) Sujet>COD>COI>Obl.>Gén.

( $\mathrm{CO}=$ =Complément d'objet direct $; \mathrm{COI}=$ Complément d'objet indirect $;$ Obl. =oblique ;

Gén. =Génitif)

NB : Le cas oblique désigne un cas grammatical autre que celui ou ceux servant de forme de citation (généralement les cas nominatif et accusatif).

Cette hiérarchie a été utilisée par Dan Maxwell (1982: 140) pour prédire les généralisations diachroniques suivantes (DG) (31) :

(31)

DGIIa : No strategy may skip any position(s) when spreading across the Accessibility

Hierarchy (AH) ;

DGIIIa : No strategy may skip any position(s) while receding along the AH ;

DGV : Two strategies in a given language tend to complement each other; as one advances, the other recedes.

DGVI : The form of a new relative clause strategy in a given language is severely restricted by the nature of the strategies already present. The new strategy must to a certain extent share syntactic features with those already present, since the former is diachronically derived from the latter.

(Maxwell 1982:140)

\subsection{Paramètres en jeu dans l'expansion des relatives en wh-}

\subsubsection{Pronoms relatifs et fonctions syntaxiques}

28 Le paramètre le plus pertinent pour identifier l'expansion des relatifs en wh- est la fonction syntaxique du relatif d'après la Hiérarchie d'Accessibilité de Keenan \& Comrie. Il est généralement admis que les relatifs en wh- font leur entrée dans la langue anglaise par le bas de la hiérarchie, c'est-à-dire par les fonctions les moins accessibles, sans doute parce que ces fonctions étaient les premières à être abandonnées par les relatifs déictiques en vieil-anglais.

Les résultats (Suarez-Gomez 2008) montrent que les relatifs pronominaux en vieilanglais et au début du moyen-anglais abandonnent les positions les plus hautes de la hiérarchie plus tard que les plus basses : les pronoms relatifs en se commencent leur récession à partir des positions les plus basses de la hiérarchie. Les résultats du début 
du moyen-anglais semblent confirmer que les pronoms en wh- sont plus souvent utilisés dans la fonction oblique ( $85 \%)$ que dans la fonction sujet (14\%). A la fin du moyen-anglais, les pronoms relatifs en wh- montrent encore une préférence pour la fonction oblique ( $45 \%$ ) sans oublier la fonction sujet ( $33 \%$ ), la position la plus haute de la hiérarchie, représentée le plus par which, qui est aussi bien utilisé pour des antécédents animés qu'inanimés.

L'exemple le plus connu de which avec un antécédent animé vient de The Lord's Prayer (32) :

(32) Our father, which art in heaven...

31 Un tiers des occurrences en which est utilisé avec un antécédent humain au $16^{\text {ème }}$ siècle mais pour le $17^{\text {ème }}$ siècle, le pourcentage tombe à dix pour cent (CHEL III, p. 294). Le changement est mis au crédit de la systématisation de l'emploi des formes grammaticales variées au cours du début de l'anglais moderne, ainsi qu'à l'expression polie de la société Tudorienne et Stuartienne, qui citait explicitement le référent de la personne (CHEL III, p. 294).

\subsubsection{Pronoms relatifs et types de subordonnée relative}

Un autre paramètre à prendre en compte dans la distribution des relatifs est le degré de restrictivité de la subordonnée relative, qui distingue les subordonnées relatives restrictives des subordonnées relatives non-restrictives. Olga Fischer (2007 : 301-305) mentionne que les pronoms en wh- apparaissent d'abord dans les subordonnées relatives non-restrictives puisque ce type de subordonnée était le locus préféré des relatifs pronominaux en vieil-anglais. Les résultats en moyen-anglais (Suarez-Gomez 2008) montrent aussi que le degré non-restrictif est le locus favori des relatifs pronominaux en wh-, surtout pour la période 1350-1420 (75\%).

33 Pour résumer, les résultats montrent que les mots en wh- en tant que pronoms relatifs se comportent comme un «bouche-trou » fonctionnel après la disparition progressive de se. Les pronoms relatifs en wh- sont favorisés dans les contextes où se en vieilanglais était préféré.

Quelles sont les conséquences de l'introduction des mots en wh- pour se et sepe? L'introduction de nouveaux membres dans le paradigme des pronoms relatifs, ici les mots en wh-, provoque la perte complète des vieux pronoms relatifs, à savoir les pronoms en se, qui déclinaient déjà. Le processus de disparition est graduel mais complet. L'élimination d'un membre du paradigme est justifiée par des principes généraux de changement linguistique, grâce auxquels les langues évitent la synonymie, en particulier la synonymie fonctionnelle.

\section{Raisons de l'apparition de do}

Le troisième point que j'aimerais souligner est l'apparition de do (voir exemple $1.21 \mathrm{du}$ texte en anglais contemporain).

Le développement historique de la périphrase do a été amplement documenté dans la littérature. Kroch (1989) a offert une analyse détaillée des données d'Ellegård (1953) pour démontrer que do, en support, s'est développé dans chacun des contextes dans 
lequel il apparaît actuellement en anglais contemporain, à la même vitesse, mais à des étapes différentes.

La transition de $\mathrm{V} 2^{1}$ à S-inversion de l'auxiliaire est à mettre au crédit de plusieurs changements cruciaux. Le premier est ce que Kiparsky appelle la licence positionnelle (" positional licencing») du sujet, qu'on exprimera en termes de règle de correspondance SUJET. Kiparsky (1996: 461) démontre qu'il y a une connexion claire entre la montée d'une position fixe pour le sujet et la perte de la marque explicite casuelle : qu'il y a une relation entre la perte de la morphologie flexionnelle et le développement de contraintes positionnelles rigides apparait clairement dans la syntaxe comparative. Le point le plus important à propos de ce lien est que ce n'est pas une vague corrélation ou tendance mais une implication sans exception, qui va dans une direction seulement (citation (33)) :

(33)

"Lack of inflectional morphology implies fixed order of direct nominal arguments (abstracting away from $\bar{A}$-movement of operators). Every Germanic language which has lost case and agreement morphology, whether VO (English, Swedish, Danish, Norwegian) or OV (Dutch, West Flemish, Frisian, Afrikaans), has imposed a strict mutual ordering requirement on its nominal arguments, without changing the headedness of its VP. The order is always that subjects precede objects, and indirect objects (NPs, not PPs) precede direct objects."

(Kiparsky 1996 : 461)

Un second changement est la formation de la sous-classe des verbes modaux qui n'a pas l'inflexion de l'accord au présent (et au passé). C'est une caractéristique distinctive de l'anglais moderne. Le développement de cette sous-classe est bien documenté (voir par exemple Denison 1993, ou plus récemment, Bybee 2006). Les changements qui sont survenus dans la transition vers l'anglais moderne étaient syntaxiques, sémantiques et morphologiques:

- les modaux ont perdu la capacité de prendre des compléments non-infinitivaux et ils ont perdu leur sens d'origine. Par exemple, cunnan ne voulait pas dire 'be able to' mais 'know', et willan voulait dire 'wish, want';

- les modaux ont acquis des sens épistémiques, ayant à voir avec la possibilité et la nécessité ;

- les modaux ont acquis la contraction de not, à savoir n't. Selon Denison (1993 : 309) ; la première occurrence date du $15^{\text {ème }}$ siècle ;

- les modaux ont perdu toute flexion de la personne ;

- l'infinitif a perdu sa flexion.

Puisque, en tant que verbes principaux, les modaux infléchis fonctionnaient comme la tête de S même en vieil-anglais, on ne trouve pas de changement dans leur comportement pour ce qui est de l'ordre des mots dans les questions et les phrases négatives.

Il est à remarquer que Warner (1993) offre de nombreux exemples en vieil-anglais d'ellipse avec les modaux et les verbes auxiliaires (34) :

(34)

Wenst pu poet se godcunda anweald ne mihte afyrran

think you that the heavenly power ne could take-away

pone anweald Jam unrihtwisan kasere, ... gif he wolde?

The empire that righteous Coesar ,... if he would?

Gise, la, gese; ic wat poet he mihte, gif he wolde.

Yes, O yes, I know that he could, if he would! 
41 Si on argue que l'ellipse n'est possible que si le verbe gouvernant est un auxiliaire, de telles données suggèreraient que les modaux étaient déjà une classe distincte de verbes en vieil-anglais (voir Warner 1993 : 113 pour la discussion). Warner soutient aussi qu'en vieil-anglais déjà, il y avait le pseudo-gapping permettant au verbe principal d'être omis, mais non l'auxiliaire ou une portion du VP. Soit (35) :

(35)

Se pe wille godcundne wisdom secan ne moeg he

He who will heavenly wisdom seek-INF ne may he

hine wip ofermetta

it with arrogance

'He who will seek heavenly wisdom may not [seek] it with arrogance.'

(Warner 1993 : 114)

\section{XSV au début de l'anglais moderne (voir Tableau 1) :}

\begin{tabular}{|l|l|l|l|}
\hline Ordre des mots & $\begin{array}{l}\text { I : } 1480-1530 \\
\text { Nombre \% }\end{array}$ & $\begin{array}{l}\text { II }: 1580-1630 \\
\text { Nombre \% }\end{array}$ & $\begin{array}{l}\text { III : } 1680-1730 \\
\text { Nombre \% }\end{array}$ \\
\hline XSV & 402480,0 & 433881,5 & 460091,2 \\
\hline XVS & 100920,0 & 98718,5 & 4448,8 \\
\hline Total & 5033100,0 & 5325100,0 & 5044100,0 \\
\hline
\end{tabular}

Tableau 1. Fréquence des ordres de mots inversés et non-inversés.

D'après le Tableau 1, il y a une perte progressive de V2 de la période I à la période III et Baekken (2000) montre aussi que V2 était sensible au type d'item lexical en position initiale.

Tels étaient les trois arguments qui ont favorisé l'apparition de l'auxiliaire do, et en particulier dans les questions.

\section{Mouvement en wh- et statut discursif dans l'histoire de l'anglais}

47 Le quatrième point abordé concernera wh- et son statut discursif dans l'histoire de l'anglais. 

l'hypothèse selon laquelle la fonction des modèles autres que ceux obéissant à l'ordre sujet-verbe-complément d'objet est d'accommoder les compléments d'objet qui ne sont pas nouveaux ou les sujets qui ne sont pas donnés dans des positions syntaxiques particulières dans l'énoncé. L'étude est fondée sur les assomptions suivantes: (i) la variation thématique joue un rôle significatif dans la grammaire de la langue et (ii) la structure informationnelle est un facteur déterminant dans la structuration formelle des phrases (Lambrecht 1994: 3). En ce qui concerne la variation thématique, l'approche fonctionnelle systémique fonde le degré de marque d'une phrase déclarative sur sa capacité à dévier d'un ordre sujet-verbe-complément d'objet non marqué. Par conséquent, le sujet d'une phrase déclarative est le thème non marqué et les autres arrangements de ces constituants principaux, par exemple, dans la thématisation, l'inversion, la dislocation à gauche, le clivage (clefting) conduisent à des modèles marqués.

\subsection{La thématisation dans l'histoire de l'anglais}

51 La thématisation était déjà utilisée en vieil-anglais (36) :

(36) Đet mynster seo ilce cwen mid hire were Ælpelrede swiðe lufade

That monastery, the queen with her husband Ethelred loved very much.

(cocathom2, ÆCHom_II_4 :33.122.750)

Deux modèles (Tableau 2) décrivent la thématisation en fonction de la structure informationnelle dans l'histoire de l'anglais.

(i) L'expression thématisée est courte (2-4 mots), ce qui indique que le poids final entre en jeu, et l'expression est localement donnée ; c'est-à-dire qu'elle est anaphorique dans le domaine discursif d'une phrase prototypique, qui est favorisé par donné-nouveau. Ce modèle, qui est d'un point de vue statistique prototypique, est prédominant de 1570 à 1640 dans le genre scientifique et le genre des procès.

(ii) L'expression topicalisée est longue (de 10 à 20 mots) et porte une information nouvelle. Ce modèle n'est pas en accord avec le poids final ou le gradient donné- 
nouveau et il est distribué par les données scientifiques, en particulier à partir de 1640-1710, et plutôt moins explicitement dans les textes de procès.

\begin{tabular}{|l|l|}
\hline Genre Période & \% Donné \\
\hline Science P1 & 25 \\
P2 & 75 \\
P3 & 90 \\
P4 & 50 \\
P5 & 100 \\
\hline Procès P1 & 25 \\
P2 & 25 \\
P3 & 100 \\
P4 & 50 \\
P5 & 75 \\
\hline
\end{tabular}

(P1 : 1500-1570 ; P2 : 1570-1640 ; P3 : 1640-1700 ; P4 : 1710-1725 ; P5 : 1750-1759)

Tableau 2. Statut discursif des NPs thématisés (d'après Pérez-Guerra 2012)

53 Puisque le modèle (i) est prototypique à la fois dans les statistiques et dans la théorie, il doit être considéré comme non marqué comparé au modèle (ii), et par conséquent, on le trouve facilement dans les données d'anglais oral.

\subsection{Constructions prédicatives existentielles}

54 Selon l'OED, les premières occurrences de prédication existentielle datent du $15^{\text {ème }}$ siècle (37-40) :

(37) There weren that dyd not so

(14) Why I can't be a Nun 244 in Early Eng. Poems \& Lives Saints (1862) 144)

(38) There were that saied, that this ambassador should be chastised

(a1533 Ld. Berners tr. A. de Guevara Golden Bk. M. Aurelius (1537) sig. Kliv)

(39) There are of them whiche accomptee it a greate aoffence to touche monie.

(1569 J. Sanford tr. H. C. Agrippa of Vanitie Artes \& Sci. 101b)

(40) There are, to whom Death doth seeme no more then a blood-letting.

(1628 O. Felltham Resolves : $2^{\text {nd }}$ Cent. Xiii. Sig. K2)

Les données (Tableau 3) montrent la nature non-anaphorique des sujets post-verbaux dans les constructions prédicatives existentielles, puisque moins de $30 \%$ des NPs examinés sont assimilés à de l'information donnée dans les deux genres. Cela va de pair avec les résultats de Birner \& Ward (1993 : 27) pour l'anglais contemporain.

Genre Période \% Information donnée 


\begin{tabular}{|l|l|}
\hline Science P1 & 12 \\
P2 & 21,9 \\
P3 & 10,7 \\
P4 & 14,3 \\
P5 & 33,3 \\
\hline Procès P1 & 10,5 \\
P2 & 4,1 \\
P3 & 22,5 \\
P4 & 16,2 \\
P5 & 32,7 \\
\hline
\end{tabular}

Tableau 3 : Statut discursif des noms postverbaux dans les prédications existentielles (d'après Pérez-Guerra 2012)

\section{Les questions tags dans l'histoire de l'anglais}

Le cinquième point que j'aimerais illustrer est les questions tags dans l'histoire de l'anglais (1.95 en anglais contemporain dans le texte).

Bien que les questions tags puissent exprimer des requêtes authentiques d'information, elles ont typiquement toute une palette de fonctions pragmatiques supplémentaires dans la conversation. Par exemple, les tags de ponctuation sont utilisés pour souligner ce que l'énonciateur dit et n'implique pas de réaction ou de réponse de la part du coénonciateur. Par conséquent, les questions tags sont souvent utilisés à l'intérieur d'un tour de parole. D'autres types pragmatiques sont représentés par les tags de confirmation, qui expriment le fait que l'énonciateur n'est pas sûr de ce qu'il dit, et par les tags péremptoires et agressifs, qui sont employés pour fermer une discussion ou provoquer et insulter les autres énonciateurs (voir Algeo 1988, 1990). Les questions tags sont donc multi-dimensionnels.

Selon Hoffmann (2006), le premier question tag est apparu en 1497. Soit (41):

(41) Than thay have some maner gettynge By some occupacione, have thay?

(Henry Medwall : Fulgens and Lucrece, 1497)

La deuxième occurrence la plus vieille est identifiée dans la collection de théâtre anglais (English Drama Collection) dans une pièce anonyme de la période Tudor, Iacob and Esau, qui fut jouée au début des années 1550. Pour la période 1550-1574, 7 occurrences furent trouvées dans des pièces. Cent vingt-deux autres exemples furent amassés dans des pièces écrites entre 1575 et 1599, ce qui atteste du fait que les marqueurs considérés deviennent pleinement établis dans le système communicatif du début de l'anglais moderne pour la fin du $16^{\text {ème }}$ siècle.

Hoffmann (2003) montre que l'emploi des questions tags fut relativement stable pendant 200 ans (c'est-à-dire entre 1550 et 1750) puis, qu'après 1750, il connut une croissance spectaculaire en fréquence : avec 428 exemples par million de mots, le début du $20^{\text {ème }}$ siècle contient 7 fois plus de questions tags que les pièces de la période Tudorienne et Elisabéthaine. Cependant, ce chiffre est relativement bas quand il est comparé aux résultats des conversations de nos jours, tels qu'ils apparaissent dans la 
section orale démographique du British National Corpus, où les questions tags ont une fréquence de 4383 par million de mots (Tottie \& Hoffman 2006).

D'autre part, la comparaison inter-genres montre aussi que la corrélation entre la formalité de la situation langagière et l'utilisation des questions tags dans l'interaction parlée tient aussi pour le théâtre anglais. Les tragédies contiennent typiquement une grande proportion de situations langagières formelles, tandis que c'est l'opposé pour le cas des comédies et autre types de textes humoristiques.

Les questions tags les plus fréquents dans le corpus sont will you? (431 occurrences, $7,3 \%$ ), is it ? (359 exemples, 6,1 \%) et do you ? (326 occurrences, 5,5\%). Près de la moitié des occurrences de will you? sont identifiées dans des tags qui sont attachés à une principale à l'impératif. Si on considère seulement les propositions principales à la forme déclarative, is it ? (357 exemples, 6,4 \%) est le plus fréquent, suivi par do you ? (326 exemples, $6,5 \%$ ) et isn't it ? (281 occurrences, 5,1\%).

Le Tableau 4 illustre les types de polarité sur la totalité des questions tags (5899) dans les pièces de la collection. Le nombre de tags à polarité constante dépasse le nombre de polarité inversée négative-positive ( $30 \%$ vs. $20,3 \%$ ). Cependant, avec tout juste un peu moins de $50 \%$ de toutes les occurrences, les questions tags à polarité positive-négative représentent une proportion bien plus petite que ceux qui apparaissent dans les pièces de Shakespeare et dans la conversation de nos jours.

\begin{tabular}{|l|l|l|}
\hline Polarité & Nombre & $\%$ \\
\hline POS-NEG & 2907 & 49,3 \\
\hline NEG-POS & 1196 & 20,3 \\
\hline POS-POS & 1772 & 30 \\
\hline NEG-NEG & 24 & 0,4 \\
\hline
\end{tabular}

Tableau 4 : Polarité des questions tags dans la Collection du Théâtre anglais

Enfin, les constructions à polarité négative-négative sont clairement un phénomène rare à travers l'histoire des questions tags. En fait, leur existence a été parfois mise en question (voir Quirk et al. 1985). Néanmoins, avec un total de 24 occurrences dans les données de Hoffmann (2006), elles sont trop fréquentes pour ne pas être mentionnées. Les exemples (42)-(44) montrent l'emploi rare dans des périodes différentes du théâtre anglais.

(42) yes, Sir, but, you see, he has not appear'd, may not I put up my rapier now, and go home again with my honour, may I not? (William Clark, MarcianoI, 1662)

(43) You won't, old Pluto, won't you; then, ma'am, observe! (Frederick Reynolds, The dramatist, 1789)

(44) And he wouldn't be persuaded by the ladies, wouldn't he ? (Frank E. Emson, The Weller family, 1878)

Du point de vue de la polarité, les questions tags du $16^{\text {ème }}$ siècle sont ainsi plus proches de l'emploi actuel que de toute autre période entre les deux. Sept des 10 questions tags les plus fréquents aux $16^{\mathrm{ème}}$ et $17^{\mathrm{ème}}$ siècles correspondent aux types les plus fréquents utilisés en anglais contemporain. 


\begin{tabular}{|l|l|l|l|}
\hline Question tag & $\begin{array}{l}\mathbf{1 5 5 0 - 1 6 4 9} \\
\text { Nb \% }\end{array}$ & Question tag & $\begin{array}{l}\text { Après 1850 } \\
\text { Nb \% }\end{array}$ \\
\hline will you & 9012,3 & isn't it & 1879 \\
\hline is it not & 7910,8 & is it & 1637,8 \\
\hline are you & 527,1 & will you & 1135,4 \\
\hline are you not & 435,9 & do you & 914,4 \\
\hline is it & 354,8 & is it not & 884,2 \\
\hline do you & 334,5 & won't you & 723,5 \\
\hline will you not & 314,2 & are you & 623 \\
\hline do you not & 294 & don't you & 572,7 \\
\hline is he not & 243,3 & ain't it & 432,1 \\
\hline did you not & 212,9 & have you & 422 \\
\hline
\end{tabular}

Tableau 5. Les question tags les plus fréquents pour 1550-1649 et après 1850 dans la Collection du Théâtre anglais

Il est difficile d'émettre une explication sur les raisons sous-jacentes au développement illustré par le Tableau 5. On pourrait avancer que la réponse se trouve dans les différentes fonctions pragmatiques des questions tags avec les différents types de polarité. Ainsi, si on regarde de plus près la période 1750-1799, période où les tags à polarité constante sont les plus fréquents, cela révèle qu'il existe un nombre important de tags à polarité constante qui expriment une attitude adversative de la part de l'énonciateur. A l'inverse, les tags à polarité inversée ne montrent pas ce genre d'attitude à ce point. Voici des exemples typiques de tags à polarité constante en (45)(47) :

(45) Oh! What, you are asleep, are you ? - I'll waken you, with a vengeance. (Knocks with his heel)

(Isaac Bickerstaff, 'Tis well it's no worse, 1770)

(46) I know you, mammon! You will tell a different tale tomorrow. I'm a coxcomb, am I ? I'll punish you!

(Thomas Holcroft, Love's frailties, 1794)

(47) What, dost thou dare to appear before me with that serpent's tongue of thine, sloughed over with lies? You dare to bring your stories to me, do you? (shaking him violently by the collar)

(Joanna Baillie, The election, 1798)

En anglais contemporain, les questions tags de type agressif, de quelque polarité que ce soit, sont une catégorie marginale ; ils représentent seulement $1 \%$ de tous les questions tags de la section étudiée du BNC (Tottie \& Hoffmann). Ainsi, on pourrait spéculer que la perte de cette fonction pragmatique particulière a pu contribuer à une décroissance de la fréquence des tags à polarité constante durant le $19^{\text {ème }}$ et le $20^{\text {ème }}$ siècles. Seule une étude diachronique détaillée pourrait confirmer cette hypothèse ou non. 
68 En ce qui concerne leur forme syntaxique, la proposition incluant les questions tags avec une polarité positive-négative et négative-négative appartient à la catégorie des questions négatives avec des sujets pronominaux. Cependant, dans les données du $16^{\text {ème }}$ siècle, l'ordre des composants individuels est bien plus fixe que dans d'autres questions négatives. Ainsi, dans pratiquement toutes les occurrences de questions tags, la particule négative suit le sujet, comme dans l'exemple (48). Le seul exemple où not précède le sujet pronominal est donné dans l'exemple (49) :

(48) Why Iohn, thou knowest the Duke of Yorkes Gunerall must be carried into England, does

thou not?

(Anonymous (Elizabethan), The famous victories of Henry V, 1583-1588)

(49) I told you, did not I?

(John Jefferes, The bugbears, 1563)

D'après Hoffmann (2003), les premiers exemples des questions tags contenant l'enclitique n't sont trouvés dans la première partie du $17^{\text {ème }}$ siècle. Leur nombre augmente régulièrement en fréquence et représente $90 \%$ de tous les exemples au début $\mathrm{du} 20^{\text {ème }}$ siècle. Par conséquent, la proportion des questions tags contenant not en position post-sujet tombe de près de $100 \%$ à près de $10 \%$.

70 La question demeure quant à savoir s'il $\mathrm{y}$ a un lien entre l'emploi de la forme non contractée not en position pré-sujet et sa variante contractée. Par exemple, il ne serait pas raisonnable d'assumer que la version non contractée a pu permettre un accroissement de l'emploi de l'enclitique n't dans les questions tags. En effet, la première occurrence de la variante non contractée est identifiée avant l'utilisation des formes contractées, et une fois que les formes contractées sont disponibles, leur nombre excède rapidement celui des variantes non contractées. Même si c'était le cas, les fréquences sont bien trop basses pour aboutir à des conclusions fiables. Il semblerait plus probable de dire que d'autres facteurs, par exemple les traits prosodiques tels l'accentuation et le rythme (voir Brainer (1989)) sont susceptibles d'avoir eu une influence dans le choix des variantes.

\section{Diachronie des questions fermées avec des particules}

Le dernier point que j'aimerais discuter est les questions fermées en yes/no et leur grammaticalisation (l. 110 en anglais contemporain dans le texte).

Ultan (1978) identifie cinq stratégies courantes pour encoder les questions interlinguistiquement : l'intonation, les particules de question, les changements d'ordre des mots, les tags en fin de phrase, et les changements phonologiques sur les segments phonologiques finaux. Les questions fermées en yes/no ont la propriété interlinguistique fonctionnelle de chercher un commentaire sur le degré de vérité de la proposition interrogée. Dans un nombre impressionnant de cas inter-langues, Bencini (2003) montre que le marqueur interrogatif pour les questions en yes/no est dérivé de or formellement similaire à un des deux éléments dans la langue : le marqueur négatif not et le marqueur disjonctif or.

73 Le fait que les particules de question ont pour origine d'autres éléments exprimant l'incertitude et le doute va de pair avec la fonction et la pragmatique des questions. D'abord, les vraies questions demandent des informations et cela prend souvent la forme d'une hypothèse exprimée par l'énonciateur et une requête pour une 
confirmation or un refus. De plus, parce que les questions sont des actes de langage manipulatifs, elles expriment souvent des phénomènes de politesse, qui ont pour but d'affaiblir la force de la requête.

L'explication diachronique proposée par Bencini (2003) est que les particules de question dérivent de la grammaticalisation d'autres éléments grammaticaux dans des constructions interrogatives nouvelles qui sont motivées par des propriétés pragmatico-discursives des questions. Ce qui est au centre de l'explication diachronique est l'idée que la particule de question grammaticalise à l'intérieur d'une construction spécifique, et dans la position qu'elle occupe à l'intérieur de cette construction (Bybee 2006), c'est-à-dire la position de la particule de la question n'occupe pas une position particulière en réponse à un courant synchronique appelé consistance typologique.

Bencini (2003) identifie trois constructions source et cheminements de grammaticalisation résultant des différentes positions de la particule de question (voir Tableau 6).

\begin{tabular}{|l|l|l|}
\hline Construction source & grammaticalisation & Position de la particule \\
\hline S (or) not S & S not $>$ S particule & finale \\
\hline or S & or S $>$ particule & S initiale \\
\hline S or & S or $>$ S particule & finale \\
\hline
\end{tabular}

Tableau 6. Constructions source et cheminements de grammaticalisation $S=$ Sentence

\subsection{La construction source interrogative offrant une alternative} «Are you coming or are you not coming ?' aussi bien que dans la forme tronquée " Are you coming or not?». L'essence de l'explication diachronique est que la particule de question est le résultat de la réanalyse de la particule négative dans le contexte d'une interrogative alternative réduite, dans laquelle la seconde partie de la question est omise. Ce processus est illustré dans (50):

(50) Are you going (or) not going > Are you going (or) not ?

NEG NEG/Particule de question

Le processus en (50) est idéalisé en ce qu'il présente seulement les étapes initiale et finale de la grammaticalisation. La grammaticalisation peut mettre en jeu une ou plusieurs étapes intermédiaires et c'est en cela que les langues pour lesquelles la grammaticalisation est en cours offrent des preuves importantes pour la documentation du processus entier (Croft 2002). De plus, le lien entre la particule de question et le marqueur négatif peut être ou non transparent synchroniquement. A l'autre bout du processus de grammaticalisation, le marqueur négatif d'origine peut avoir entièrement perdu son lien avec la négation dans l'état synchronique de la langue. L'explication diachronique de Bencini n'empêche pas un rôle important de la 
synchronie. Contrairement aux explications typologiques anciennes, cependant, ce sont les propriétés pragmatico-discursives des questions, et non la consistance typologique, qui sont la force dominante derrière l'émergence des différentes constructions source. Bencini (2003) propose que ces propriétés soient décrites de la façon suivante. Soit (51) :

(51) Propriétés pragmatico-discursives de l'interrogative alternative

i. Présupposition : les interrogatives alternatives présupposent quelque chose avant le partage du contenu de la proposition.

ii. l y a un contraste entre les propositions qui se contredisent : cela peut conduire à de l'urgence ou de l'impatience.

\subsection{La construction source or $S$}

81 La construction or $S$ a la forme pragmatique d'une question qui est ancrée dans le contexte précédent. L'énonciateur peut continuer une partie de la question précédente en suggérant des alternatives à la proposition exprimée dans la proposition initiale (52-54).

82 Selon l'OED, les premières occurrences datent du début du $20^{\text {ème }}$ siècle :

(52) I suppose the matter is technically your concern as well as mine, or is it?

(c1907, W. B. Yeats, Let. 4 July (1954) 483)

(53) Matilda had the laugh of the bees after all. Or did she? I'm not so sure!

(1924, R. Macaulay, Orphan Island xvi. 212)

(54) They couldn't have come from the servants' quarter or you'd have heard. Or would

you? You were dropping off.

(1935, V. Markham, Deadly Jest xi. 138)

\subsection{La construction source $S$ or}

83 La meilleure façon de décrire la construction $S$ or est de dire que c'est la marque de l'ouverture grammaticalisée. L'énonciateur indique qu'il y a une palette d'alternatives dont le co-énonciateur peut se servir mais il ne spécifie pas lesquelles. Contrairement à l'interrogative alternative, il n'y a pas de contraste. Les occurrences ne sont pas présentes dans l'OED mais on en trouve en anglais américain. La langue anglaise a une version optionnelle de cette construction, dont les exemples abondent dans la conversation en anglais américain (55-56):

(55) That's interesting. Did it, did it work out pretty well in the end ? mean, did a couple of people sort of gradually, sort of assume, uh sort of a moderator role in the trial or?

(Switchboard, file 4036)

(56) ...Well, pottery sounds interesting have you mad a lot of, uh, a lot of vases and things or?

(Switchboard, file 2045)

\section{Conclusion}

Pour conclure, la perspective diachronique envisagée dans cet article nous a permis de souligner quelques faits saillants sur les questions et de mieux comprendre comment la diachronie peut éclairer des points synchroniques soulevés dans le texte d'anglais contemporain dûment choisi et qui sont représentés par les pronoms relatifs en wh-, 
l'auxiliaire do, les syntagmes nominaux thématisés et les prédications existentielles, les questions tags et les questions ouvertes et fermées.

\section{BIBLIOGRAPHIE}

Algeo, J. (1988). The Tag Questions in British English ; it's different I'n'it ? English World-Wide 9 : 171-191.

Algeo, J. (1990). It's a Myth Innit ? Politeness and the English Tag Question. In Ricks, O.\& Michaels, L.. The State of the Language. Berkeley : University of California Press.

Brainerd, B. (1989). The contractions of not : a historical note. Journal of English Linguistics 22(2) : 176-204.

Baekken, B. (2000). Inversion in Early Modern English. English Studies 5 : 593-421.

Bencini, G. (2003). Towards a diachronic typology of yes/no question constructions with particles. Chicago Linguistics Society 39 : 604-621.

Birner, B. \& Ward, G. (1993). There-sentences and inversion as distinct constructions : a functional account. In Proceedings of the $19^{\text {th }}$ Annual Meeting of the Berkeley Linguistics Society, 27-39. Berkeley: Berkeley Linguistics Society.

Bybee, J. (2003). Mechanisms of change in grammaticization : the role of frequency. In Janda, R. \& Joseph, B. D. (eds.). Handbook of Historical Linguistics Oxford : Blackwell. 602-623.

Bybee, J. (2006). Frequency of use and the organization of verbal inflection. Oxford : Oxford University Press.

Croft, W. (2002). Typology and Universals ( $2^{\text {nd }}$ edition). Cambridge : Cambridge University Press.

Denison, D. (1993). English Historical Syntax. London \& New York : Longman.

Ellegård, A. (1953). The auxiliary do : The establishment and regulation of its use in English. Stockholm : Almqvist \& Wiksell.

Fischer, O. (2007). Morphosyntactic change. Functional and formal properties. Oxford : Oxford University Press.

Hoffmann, S. (2006). Tag questions in Early and Late Modern English : Historical description and theoretical implications. Anglistik 17 : 35-55.

Keenan, E. L. \& Comrie B. (1977). Noun phrase Accessibility and Universal Grammar, Linguistic Inquiry 8 (1) : 63-99.

Kiparsky, P. (1996). The shift to head-initial VP in Germanic. Studies in comparative Germanic syntax II, ed. by H. Thrainsson, S. D. Epstein \& S. Peter, 140-179. Dordrecht : Kluwer Publishers.

Kroch, A. (1989). Amount qualification, referentiality, and long wh- movement. Manuscrit non publié. University of Pennsylvania.

Lambrecht, K. (1994). Information Structure: Topic, Focus, and the Mental Representations of Discourse Referents. Cambridge : Cambridge University Press. 
Maxwell, D. (1982). Implications for NP accessibility for historical syntax. Folia Linguistica Historica $3(2): 135-152$.

Pérez-Guerra, J. (2012). Discourse status and syntax in the history of English. In Meurman-Solin, A., Lopez-Couso, M.-J. \& Los, B., Information structure and syntactic change in the history of English. Oxford : Oxford University Press. 121-138.

Prince, E. F. (1979). On the given/new distinction. In Hanks, W. F., Clyne, P. R. \& Hofbauer, C. L. Papers from the $15^{\text {th }}$ Regional Meeting of the Chicago Linguistics Society, 267-325. Chicago : Chicago Linguistics Society.

Quirk, R. et al. (1985). A Comprehensive Grammar of the English Language. London : Longman. Suarez-Gomez, C. (2008). Strategies in competition : demonstratives and interrogatives as relativizers in the history of English. English Studies 89 (3) : 339-350.

Tottie, G. \& Hoffmann S. (2006). Tag Questions in English in British and American English. Journal of English Linguistics 34(4) : 283-311.

Ultan, R. (1978). Some general characteristics of interrogative systems. In Shopen T. (ed.). Language Typology and syntactic description. vol. 1. Cambridge : Cambridge University Press.

Warner, A. (1993). English auxiliaries. Cambridge : Cambridge University Press.

\section{Corpus}

Complete corpus of Old English [Electronic resource] : the Toronto dictionary of Old English corpus. Toronto : The University of Toronto Centre for Medieval Studies.

\section{NOTES}

1. Une langue V2 est une langue qui place les verbes finis (c'est-à-dire conjugués) après un constituant initial, souvent un adverbe. Le sujet peut se situer soit avant, soit après le verbe fini (voir The Cambridge History of the English Language, vol. 1, p. 275).

\section{RÉSUMÉS}

La thématique des questions d'un point de vue diachronique est l'objet de cet article. Dans un premier temps, est proposé un tour d'horizon des questions ouvertes et fermées depuis le vieil anglais avant de nous intéresser à l'apparition et à l'évolution des pronoms relatifs en wh-. L'auxiliaire do est brièvement discuté. Sont ensuite analysés les syntagmes nominaux thématisés et les prédications existentielles, puis les questions tags ainsi que la grammaticalisation des questions fermées.

The topic of questions from a diachronic point of view is the object of this article. First, we are interested in the study of open and closed questions since old English before moving to the discussion of the appearance and evolution of relative pronouns in wh-. The auxiliary do is briefly discussed. Then we focus our attention on topicalised nominal syntagms and existential predications then questions tags before finishing on the grammaticalisation of closed questions. 
INDEX

Keywords : questions, relative pronouns, do, questions tags, diachrony.

Mots-clés : questions, pronoms relatifs, do, questions tags, diachronie

\section{AUTEUR}

SYLVIE HANCIL

Université de Rouen

Equipe ERIAC

hancilfr@yahoo.fr 